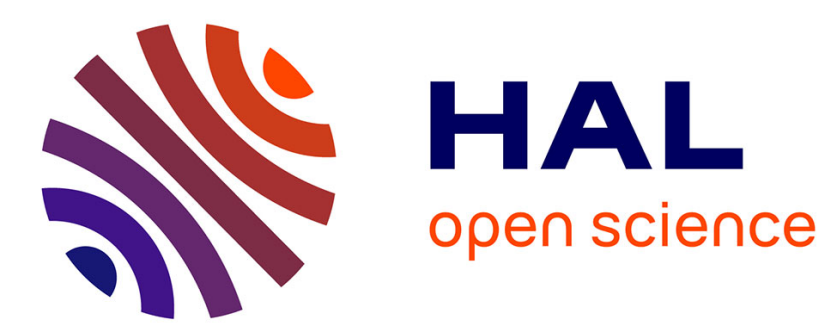

\title{
Composition lipidique d'un sous-produit de lactosérum de caséinerie
}

\author{
V Ducruet, C Degas, G Lamberet
}

\section{To cite this version:}

V Ducruet, C Degas, G Lamberet. Composition lipidique d'un sous-produit de lactosérum de caséinerie. Le Lait, 1990, 70 (2), pp.117-126. hal-00929193

\section{HAL Id: hal-00929193 \\ https://hal.science/hal-00929193}

Submitted on 1 Jan 1990

HAL is a multi-disciplinary open access archive for the deposit and dissemination of scientific research documents, whether they are published or not. The documents may come from teaching and research institutions in France or abroad, or from public or private research centers.
L'archive ouverte pluridisciplinaire HAL, est destinée au dépôt et à la diffusion de documents scientifiques de niveau recherche, publiés ou non, émanant des établissements d'enseignement et de recherche français ou étrangers, des laboratoires publics ou privés. 


\title{
Composition lipidique d'un sous-produit de lactosérum de caséinerie
}

\author{
V Ducruet 1, C Degas 2, G Lamberet 2 \\ 1 INRA, Laboratoire des sciences de la consommation, 78350 Jouy-en-Josas; \\ 2 INRA, Laboratoire de recherches laitières, 78350 Jouy-en-Josas, France
}

(Reçu le 9 août 1989; accepté le 20 décembre 1989)

\begin{abstract}
Résumé - La composition lipidique d'une poudre, sous-produit de la caséinerie, et résultant de la délipidation du lactosérum, a été déterminée par extraction par solvants, filtration sur Célite 545 , et séparation par chromatographie sur couche mince. Les acides gras sont déterminés par chromatographie en phase gazeuse après méthylation. Cette poudre contient $8,3 \%$ de lipides dont $35 \%$ sont des lipides polaires : phospholipides et céramides héxosides. Les phospholipides, qui représentent $66 \%$ de cette fraction, se répartissent de la façon suivante : phosphatidylcholine $(36,6 \%)$, sphingomyéline $(36,8 \%)$, phosphatidyléthanolamine $(13,2 \%)$, phosphatidylsérine $(11,8 \%)$ et phosphatidylinositol $(1,4 \%)$. La fraction céramide héxoside (34\% des lipides polaires) est composée pour $50 \%$ d'acides gras longs et saturés. Ces céramides mono- et di-héxosides semblent être sélectivement augmentés par le processus technologique d'ultrafiltration.
\end{abstract}

caséinerie / ultrafiltration / lactosérum / phospholipides / glycolipides / acides gras

Summary - Lipid composition of a byproduct issued from whey of casein industry. The lipid composition of a powder, as a by-product of casein industry and obtained from delipidation of whey, was evaluated by solvents extraction, filtration through Celite 545 and separation by thin layer chromatography. Fatty acids were determined by gas chromatography after methylation. The powder contained $8.3 \%$ of lipids of which $35 \%$ were polar lipids: phospholipids and ceramideshexosides. Phospholipids represent $66 \%$ of this fraction. Their distribution is as follows: phosphatidylcholine (36.6\%), sphingomyelin (36.8\%) phosphatidylethanolamine (13.2\%), phosphatidylserine (11.8\%) and phosphatidylinositol (1.4\%). The ceramides-hexosides fraction (34\% of polar lipids) contains $50 \%$ of long and saturated fatty acids. These ceramides mono- and di-hexosides seem to be selectively increased by the technological process of ultrafiltration.

casein industry / ultrafiltration / whey / phospholipids / glycolipids / fatty acids

\section{INTRODUCTION}

Le fractionnement des protéines du lactosérum sur membrane d'ultrafiltration s'est fortement développé dans l'industrie laitière à partir des années 70 . Cette séparation conduit à l'obtention d'une large gamme de fractions protéiques aux pro- priétés fonctionnelles particulièrement intéressantes pour l'industrie alimentaire : forte rétention de l'eau, pouvoir gélifiant, solubilité à tout $\mathrm{pH}$ et pouvoir moussant.

Au cours du fractionnement, des lipides résiduels issus de la membrane du globule gras sont concentrés à la même vitesse que les protéines. Ces lipides présentent 
un effet négatif sur le processus de séparation : de par leurs propriétés amphiphiles, ils s'adsorbent sur les membranes d'ultrafiltration et conduisent rapidement à un colmatage des pores mais ils jouent aussi un rôle dépresseur sur les propriétés fonctionnelles des concentrats protéiques (pouvoir moussant par exemple) (Maubois et al, 1987).

Fauquant et al (1985) ont mis à profit les propriétés agrégantes de ces complexes lipoprotéiques, en présence d'ions calcium, sous l'effet d'un léger traitement thermique $\left(55^{\circ} \mathrm{C}, 8 \mathrm{~min}\right)$, à $\mathrm{pH} 7,3$, pour développer un procédé de séparation. Les agrégats lipoprotéiques sont séparés du lactosérum clarifié par microfiltration tangentielle sur membrane.

Nous avons cherché dans cette étude à caractériser la fraction lipidique de ces complexes dans le but d'une meilleure maîtrise et compréhension du processus technologique.

\section{MATÉRIEL ET MÉTHODES}

\section{Matériel}

\section{Echantillon}

La poudre lipoprotéique (PLP) utilisée présente la composition globale suivante (Maubois, communication personnelle) : ( $\mathrm{g} / 100 \mathrm{~g}$ de poudre)

\begin{tabular}{|c|c|}
\hline Humidité & \\
\hline Protéines & \\
\hline Matières & \\
\hline $\mathrm{Ca}^{++}$ & $4,7 \%$ \\
\hline $\mathrm{Na}^{+}$ & $0,5 \%$ \\
\hline $\mathrm{K}^{+}$ & $0,3 \%$ \\
\hline $\begin{array}{l}\text { Autres } \\
\text { dont }\end{array}$ & $10 \%$ \\
\hline
\end{tabular}

\section{Solvants et réactifs}

Solvants : qualité Normapur (Prolabo), redistillés; Célite 545 (Prolabo); $\mathrm{CaHPO}_{4} \cdot 2 \mathrm{H}_{2} \mathrm{O}$ (Merck); esters méthyliques d'acides gras témoins (Sigma); phospholipides témoins (Sigma).

\section{Méthodologie}

\section{Extraction des lipides}

Les lipides de PLP sont extraits selon le protocole décrit par Marmer et Maxwell (1981), à température ambiante et en présence de $0,02 \%$ (pds/v) de BHT (fig 1); $12 \mathrm{~g}$ de PLP sont broyés au mortier en présence de $10 \mathrm{~g}$ d'un mélange de $\mathrm{CaHPO}_{4}$-Célite 545 (9:1, pds/pds) puis déposés dans une colonne en verre $(3,5 \times 30 \mathrm{~cm})$ dont la partie inférieure a été préalablement remplie de $20 \mathrm{~g}$ de $\mathrm{Na}_{2} \mathrm{SO}_{4}$ et de $15 \mathrm{~g}$ de Célite 545.

Dans une première étape $(A)$, les lipides sont élués de la colonne en 2 fractions : les lipides neutres par $150 \mathrm{ml}$ de chlorure de méthylène pur, les lipides les plus polaires par $100 \mathrm{ml}$ d'un mélange de chlorure de méthylène-méthanol $(9: 1, v / v)$.

Lors d'une seconde étape (B), la fraction la plus polaire est purifiée sur cartouche de silice vierge (capacité de $1 \mathrm{ml}$, Sep-pack, Waters). Une cartouche permet le traitement de $100 \mathrm{mg}$ de lipides totaux : les lipides neutres sont élués par $20 \mathrm{ml}$ de chloroforme, les lipides polaires par $30 \mathrm{ml}$ de méthanol.

\section{Séparation et dosage des lipides polaires}

Les lipides polaires sont analysés sur plaque de verre $(20 \times 20 \mathrm{~cm})$ recouverte de gel de silice $\mathrm{H}$ (type 60, Merck). Les plaques d'une épaisseur de $0,3 \mathrm{~mm}$ sont lavées avant utilisation par élution à front perdu du mélange chloroformeméthanol $(2: 1, v / v)$ puis activées une heure à $110^{\circ} \mathrm{C}$ avant dépôt des échantillons. Plusieurs systèmes de migration, décrits dans le tableau I, permettent l'analyse des fractions lipidiques.

Les lipides sont révélés par pulvérisation sur les plaques chromatographiques d'un mélange $\mathrm{H}_{2} \mathrm{SO}_{4}$-éthanol $(50: 50, \mathrm{~V} / \mathrm{v})$, puis chauffage à 
$150^{\circ} \mathrm{C}$. La présence de sucres et de l'acide sialique est recherchée par pulvérisation d'un réactif à base soit de 1-naphtol, soit de résorcinol (Christie, 1982).

Le dosage du phosphore selon Ames (1966) est effectué sur les zones de gel révélées, grattées puis transférées dans des tubes Pyrex. Les absorptions sont lues à $830 \mathrm{~nm}$, le poids moléculaire moyen des phospholipides est estimé à 25 fois le poids atomique du phosphore.

La lecture des plaques chromatographiques est réalisée, après révélation, à $500 \mathrm{~nm}$ avec un densitomètre CAMAG.

\section{Analyse des acides gras}

Les fractions lipidiques issues de la purification sur colonne ou de la séparation par chromatographie sur couche mince sont transméthylées à l'aide du mélange $\mathrm{BF}_{3}$-méthanol (14:86, v/v, Sigma). Pour la chromatographie sur couche mince, les lipides sont révélés rapidement (temps inférieur à $30 \mathrm{~s}$ ) par des vapeurs d'iode, les zones de gel correspondant aux espèces lipidiques sont grattées et mises en présence du réactif. Le mélange est chauffé à $90^{\circ} \mathrm{C}$ pendant 30 min dans des tubes Pyrex bouchés Téflon. Après lavage par $\mathrm{NaCl}$ saturé, les esters méthyliques sont extraits deux fois par de l'hexane. Pour la sphingomyéline et les céramideshéxosides, le temps de réaction est poursuivi jusqu'à $14 \mathrm{~h}$ à $90^{\circ} \mathrm{C}$.

Les acides gras méthylés sont analysés à l'aide d'un chromatographe en phase gazeuse (Girdel 300 ) couplé à un intégrateur Shimadzu CR3A, et équipé d'un injecteur on-colonne ( $\mathrm{J}$ et W, USA). La séparation s'effectue sur colonne capillaire de Carbowax $20 \mathrm{M}$ greffé chimiquement au support $(0,32 \mathrm{~mm} \times 30 \mathrm{~m}$, épaisseur de phase de $0,5 \mu \mathrm{m} ; \mathrm{J}$ et $\mathrm{W}$, USA). La séparation des esters est réalisée avec programmation de température de $45^{\circ} \mathrm{C}$ à $240^{\circ} \mathrm{C}$, à raison de $3^{\circ} \mathrm{C}$ par min. Le pourcentage des différents esters est calculé par rapport à l'aire totale des composés majoritaires du profil.

\section{RÉSULTATS ET DISCUSSION}

Les lipides extraits selon Marmer et Maxwell (1981) représentent $8,3 \%$ de PLP (moyenne de 4 déterminations, $\pm 0,5$ ).
Cette extraction a été mise en œuvre sur PLP car elle permet une bonne récupération des lipides polaires des produits laitiers, par rapport à 2 méthodes de référence : Roese-Gottlieb et Folch (Maxwell et al, 1986). Toutefois, cette technique n'assure pas une séparation directe des lipides neutres et des lipides polaires (fig 1). Ces derniers n'ont pu être isolés sélectivement qu'à l'aide d'une élution complémentaire sur colonne de silice. La qualité de la séparation est contrôlée par chromatographie sur couche mince à l'aide du système I (tableau I). Le rendement de la séparation sur Sep-pack est supérieur à $95 \%$. Le rapport pondéral des lipides neutres et des lipides polaires est de $65( \pm 0,45): 35$ $( \pm 0,56)$ dans l'extrait lipidique total de PLP.

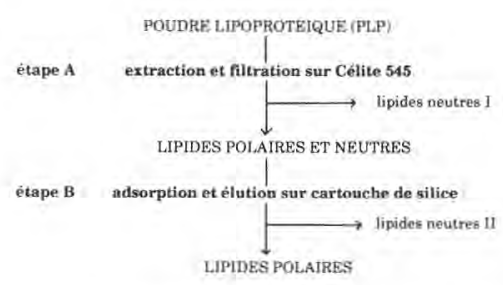

BHLAN DE LA PHEPARATION (\% ponderal par rapport aux lipides totaux de PLP): LIPIDES NEUTRES $\mathrm{I}+\mathrm{II}$ : L.IPIDES POLAIRES :

(lipides phosphorylés I lipides non phosphorylés

65 23,1
11,9

Fig 1. Schéma d'isolement des lipides polaires de la poudre lipoprotéique.

Scheme for the isolation of polar lipids from Iipoproteic powder.

\section{Analyse des phospholipides}

Le dosage du phosphore révèle que les phospholipides représentent $66 \%$ de la fraction polaire. Les différentes classes de ces phospholipides sont séparées par chromatographie sur couche mince, à l'aide du système II (tableau I). Grâce à la 
Tableau I. Systèmes de migration chromatographique sur couche mince pour l'étude des lipides de la poudre lipoprotéique (PLP). Systèmes I, II et III* : d'après Wolff et al (1984); Système IV* : d'après Skipski et Barclay (1969); Système V : d'après Morrison et al (1965). " : pour ces 2 systèmes, le front du solvant 2 est arrêté à $2 \mathrm{~cm}$ en dessous du front du solvant 1 .

Solvent systems used for lipid thin layer chromatography from lipoproteic powder (PLP). System I, II and II": from Wolff et al (1984); System IV*: from Skipski and Barclay (1969); System V: from Morrison et al (1985); ": for both systems, solvent front 2 was stopped $2 \mathrm{~cm}$ below the level of solvent front 1.

\begin{tabular}{|c|c|c|c|}
\hline Solvants & Migration & Lipides & Système \\
\hline $\begin{array}{l}\text { Hexane/éther/acide acétique } \\
80: 20: 2, v / v\end{array}$ & monodimensionnelle & lipides neutres & 1 \\
\hline $\begin{array}{l}1 . \\
\text { Chloroforme/acétone/méthan } \\
\text { acide acétique/eau } \\
75: 30: 15: 15: 7,5, v / v \\
2 . \\
\text { n-butanol/acide acétique/eau } \\
60: 20: 20, v / v\end{array}$ & bidimensionnelle & phospholipides & II \\
\hline $\begin{array}{l}1 . \\
\text { Ether/acétone } \\
90: 30, \mathrm{v} / \mathrm{v} \\
2 . \\
\text { chloroforme/méthanol/eau } \\
75: 25: 4, \mathrm{v} / \mathrm{v}\end{array}$ & $\begin{array}{l}\text { monodimensionnelle } \\
\text { à double développement }\end{array}$ & phospholipides & III \\
\hline $\begin{array}{l}1 . \\
\text { Acétone/hexane } \\
1: 3, v / v \\
2 \text {. } \\
\text { Chloroforme/méthanol/acide } \\
\text { acétique/eau } \\
25: 15: 4: 2, \mathrm{v} / \mathrm{v}\end{array}$ & $\begin{array}{l}\text { monodimensionnelle } \\
\text { à double développement }\end{array}$ & lipides polaires & IV \\
\hline $\begin{array}{l}\text { Chloroforme/méthanol/acide } \\
\text { acétique/eau } \\
65: 25: 8: 4, \mathrm{v} / \mathrm{v}\end{array}$ & monodimensionnelle & lipides polaires & V \\
\hline
\end{tabular}

grande sensibilité de la détection sur plaque associée à celle du dosage du phosphore (limite inférieure égale à $4 \mu \mathrm{g}$ de phospholipides), la répartition pondérale des différentes classes est appréciée aisément à partir d'un dépôt de $1 \mathrm{mg}$ de lipides polaires totaux (tableau II). Cette distribution est voisine de celle rapportée pour la membrane du globule gras (Christie, 1983) avec un léger enrichissement pour la sphingomyéline (SM) au détriment de la phosphatidyléthanolamine (PE).

La composition en acides gras des classes de phospholipides (tableau II) nécessite l'utilisation des systèmes chromatographiques II et III (tableau I). Le système 
Tableau II. Composition en acides gras des lipides polaires de la poudre lipoprotéique : pourcentages pondéraux des esters méthyliques des principaux acides. a : répartition pondérale, (exprimée en \%), des différentes classes de phospholipides, établie à partir du dosage de phosphore; b : PS n'a pu être obtenu suffisamment pur pour établir le profil des acides gras (détails donnés dans le texte).

Fatty acid composition of polar lipids from lipoproteic powder: relative percentage of major fatty acid methyl esters. a: ponderal distribution (expressed as \%), of different class of phospholipids, issued from phosphore determination; $b$ : PS was not sufficently pure to determinate fatty acids profile (details are given in the next).

Classes a

Acides gras

\begin{tabular}{cccccc}
\hline$P E$ & $P C$ & $P I$ & $S M$ & $P S^{b}$ & $C M H+C D H$ \\
$(13,2)$ & $(36,6)$ & $(1,4)$ & $(36,9)$ & $(11,8)$ & \\
\hline
\end{tabular}

\begin{tabular}{lrrrrr}
\hline & & & & & \\
$14: 0$ & 0,7 & 11,6 & 1,3 & 0,4 & 0,7 \\
$16: 0$ & 9,6 & 41,3 & 19,8 & 10,9 & 12,2 \\
$16: 1 n-7$ & 1,2 & 1,6 & - & - & 0,8 \\
$18: 0$ & 14,1 & 11,9 & 13,2 & 2,6 & 10,1 \\
$18: 1 n-9$ & 53,5 & 29,7 & 32,8 & 0,5 & 21,7 \\
$18: 2 n-6$ & 8,6 & 3,9 & 4,3 & 0,4 & 3,7 \\
$18: 3 n-3$ & 2,1 & - & 0,7 & - & 0,7 \\
$20: 0$ & 2,4 & - & - & 0,6 & 0,5 \\
$20: 1 n-9$ & - & - & - & 0,2 & 1,2 \\
$20: 4 n-6$ & 1,5 & - & - & 0,7 & 0,5 \\
$20: 5 n-3$ & 0,7 & - & - & 0,4 & 0,2 \\
$22: 0$ & 1,6 & - & 7,2 & 20,7 & 15,1 \\
$22: 5 n-3$ & 0,9 & - & - & - & 0,5 \\
$23: 0$ & 1,9 & - & 10,8 & 35,1 & 18,9 \\
$23: 1$ & - & - & 1,3 & 0,8 & 1,2 \\
$24: 0$ & 1,2 & - & 7,3 & 25,0 & 11,2 \\
$24: 1$ & - & - & 1,3 & 0,5 & 0,8 \\
$25: 0$ & - & - & - & 1,2 & - \\
AG saturés \% & 31,5 & 63,5 & 60,2 & 96,7 & 68,7 \\
AG insaturés \% & 68,5 & 36,5 & 39,8 & 3,5 & 31,3 \\
AG C20 à C25 \% & 10,2 & - & 27,9 & 85,2 & 50,1 \\
\hline
\end{tabular}

II assure la meilleure séparation si le dépôt n'excède pas $4 \mathrm{mg}$ de lipides polaires totaux. Cette quantité est, du fait de la répartition entre les classes, insuffisante pour déterminer les acides gras des classes minoritaires tels que la phosphatidylinositol (PI) et PE. L'évaluation des acides gras de ces 2 classes est réalisée avec le système III où le dépôt de l'échantillon s'effectue en ligne. Les acides gras de la phosphatidylsérine (PS) n'ont pu être identifiés car cette classe est mal séparée par le sys- tème III, et le système II bidimensionnel ne permet pas une collecte en quantité suffisante.

PE est la fraction la plus insaturée des 4 classes de phospholipides : l'acide oléïque contribue à lui seul pour la moitié des acides gras totaux. La phosphatidylcholine (PC) est une classe ne contenant aucun acide gras à nombre d'atomes de carbone supérieur à 18 : les 2 constituants principaux sont l'acide palmitique $(41,3 \%)$ et l'acide oléïque $(29,7 \%)$. PI présente un 
rapport acides gras saturés : acides gras insaturés voisin de celui obtenu pour PC mais sa composition s'en distingue par une proportion plus faible de $\mathrm{C} 14: 0$ et de C16:0, qui est compensée par la présence d'environ $28 \%$ d'acides gras à longue chaîne, pour l'essentiel, saturés (C22:0 à C24:0). SM est la fraction phospholipidique la plus saturée, 3 acides gras (C22:0, C23:0, C24:0) contribuent pour $85,2 \%$ à la composition totale.

Ces résultats sont en accord avec les nombreux travaux cités par Christie (1983), pour la composition en acides gras des phospholipides d'origine laitière. La proportion des acides gras hydroxylés par rapport aux acides gras totaux des sphingolipides a été estimée à moins de $1 \%$ (Morrison et Hay, 1970). Ces acides n'ont pu être révélés au niveau de SM et des céramides héxosides au cours de cette étude, la mise en évidence de cette faible fraction nécessiterait de mettre en œuvre des techniques de dérivation plus spécifique et d'identification par spectrométrie de masse.

\section{Analyse des lipides polaires non phos- phorylés}

Les lipides non phosphorylés représentent $34 \%$ des lipides polaires de PLP. Nous avons cherché à caractériser cette fraction à l'aide de plusieurs systèmes chromatographiques. Les lipides polaires, dans le système IV (tableau I), font apparaître une bande importante migrant légèrement en dessous du front de solvant. Cette zone de gel est grattée, les lipides sont élués par un mélange chloroforme-méthanol $(2: 1, v / v)$ et soumis à une nouvelle migration selon le système $V$. Deux bandes sont détectées, qui se colorent en pourpre par révélation au 1-naphtol, indiquant la présence de glycolipides.
Le comportement chromatographique de ces lipides avec le système $V$ est voisin de celui observé avec des céramides mono- et di-héxosides ( $\mathrm{CMH}$ et $\mathrm{CDH}$, respectivement) témoins, ne contenant pas d'acides gras hydroxylés (fig 2). Deux bandes sont détectées : la bande 1, de Rf égal à 0,94 , correspond à des glycocéramides $(\mathrm{CMH})$, la bande 3 observée entre $P E$ et $P C+P S$, de Rf égal à 0,69 , est attribuable à des lactocéramides (CDH). Des glucocéramides témoins (Sigma) contenant $98 \%$ d'acides gras hydroxylés migrent à une distance inférieure à celle observée pour CMH de PLP (migration non représentée).

L'ordre de migration des lipides polaires de PLP diffère légèrement de celui donné par Morrison et al (1965), avec ce système de solvants. L'inversion constatée pour PE et $\mathrm{CDH}$ rapproche nos résultats de ceux

Tableau III. Composition lipidique de la fraction polaire de PLP. a : pourcentages relatifs exprimés en $\%$ de la surface totale des pics obtenus par densitométrie à $500 \mathrm{~nm}$. Moyenne de 4 lectures. Ecart type; $b$ : pourcentages relatifs déterminés à partir du dosage du phosphore. Moyenne de 4 déterminations. Ecart type. Lipidic composition of polar fraction of PLP. a: relative percentages, expressed in $\%$ of the total area of peaks obtained by densitometry at 500 $\mathrm{nm}$. Mean of 4 evaluations. Standard deviation; $b$ : relative percentages issued from phosphore determination. Mean of 4 evaluations. Standard deviation.

Composé lipidique Densitogrammea Dosage

\begin{tabular}{lrr}
\hline CMH & $11 \pm 0,8$ & \\
CDH & $30 \pm 3,1\}$ & $34 \pm 1,70$ \\
PE & $9 \pm 0,9$ & $8,7 \pm 1,60$ \\
PS & $28 \pm 5,1$ & $7,8 \pm 0,20$ \\
PC & $24,2 \pm 2,59$ \\
SM & $22 \pm 0,9$ & $24,3 \pm 1,45$ \\
PI & - & $0,9 \pm 0,13$ \\
\hline
\end{tabular}



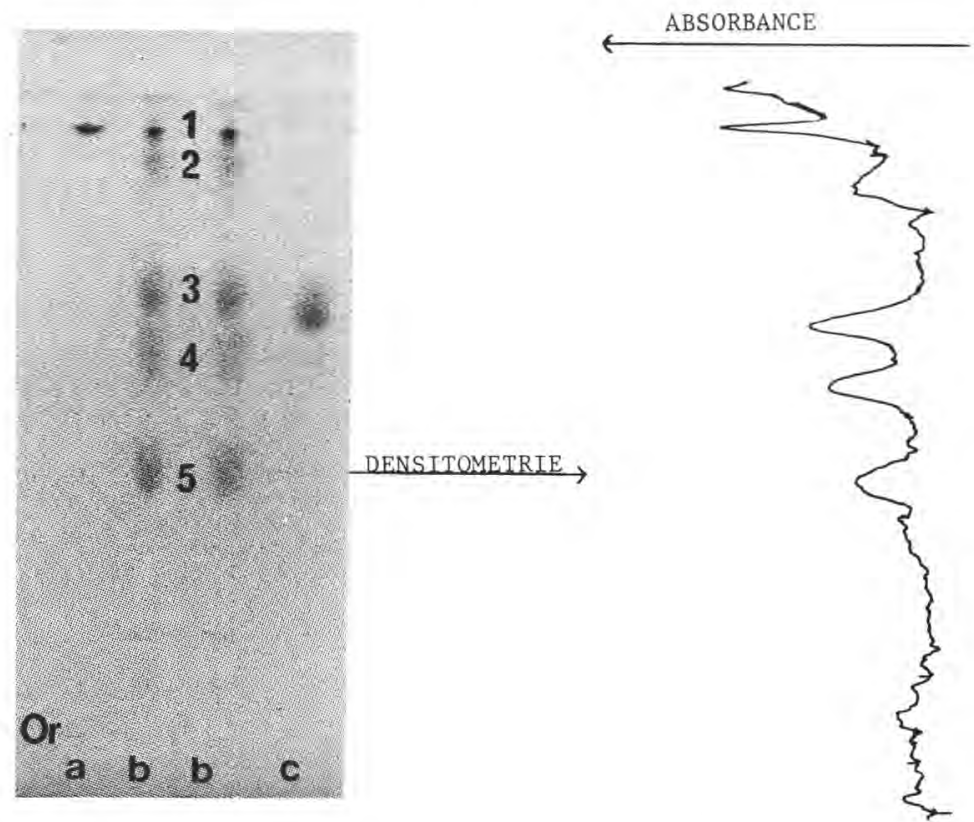

Fig 2. Chromatographie sur couche mince des lipides polaires de PLP, sur gel de silice H, développé selon le système V. Densitogramme à $500 \mathrm{~nm}$. a : galactocéramides de cerveau de bceuf ne contenant pas d'hydroxy-acides (Sigma); $b$ : lipides polaires de PLP*: $1=\mathrm{CMH} ; 2=\mathrm{PE} ; 3=\mathrm{CDH} ; 4=\mathrm{PS}$ + PC; 5 = SM; c : lactocéramides de cerveau de bœuf (Sigma); " : les glycérophospholipides de PLP sont identifiés par comparaison de leurs comportements chromatographiques par rapport à ceux de phospholipides témoins (Sigma).

Thin layer chromatrography of PLP polar lipids on $H$ silica gel developed with system V. Densitogram at $500 \mathrm{~nm}$. a: galactoceramides from deprived hydroxy acids bovine brain (Sigma); ${ }^{b}$ : PLP* polar lipids: $1=C M H ; 2=P E ; 3=C D H ; 4=P S+P C ; 5=S M$; ; lactoceramides from bovine brain (Sigma); * PLP glycerophospholipids were identified by comparing their chromatographic behaviour with authentic standards (Sigma).

obtenus par Fujishima et al (1970) avec le même mélange de migration mais ne contenant pas d'acide acétique.

La répartition des différentes classes de phospholipides établie à partir du densitogramme de la séparation réalisée par le système $V$ (fig 2) est assez voisine de celle issue du dosage du phosphore (tableau III). Les 2 fractions $\mathrm{CMH}$ et $\mathrm{CDH}$ représentent ainsi entre 34 et $40 \%$ de la fraction polaire totale de PLP. Selon le densitogramme, le rapport pondéral $\mathrm{CDH}$ $\mathrm{CMH}$ serait de 75:25.

Aucune réaction au résorcinol n'est obtenue au niveau des différents lipides polaires de PLP (fig 2), notamment pour les bandes détectées entre le dépôt et SM, zone où les gangliosides auraient été susceptibles de migrer, selon ce système de solvants. La recherche de ces composés n'a pas été plus approfondie et mériterait d'être poursuivie. 
Tableau IV. Composition en acides gras des céramides héxosides de différentes fractions d'origine laitière. Influence du type d'hydrolyse. a : selon Morrison et al (1965) : babeurre, transméthylation par $\mathrm{BF}_{3}$-méthanol 90 min à $100^{\circ} \mathrm{C}$; ${ }^{\circ}$ : selon Morrison et Hay (1965) : babeurre, saponification puis méthylation par $\mathrm{BF}_{3}$-méthanol $24 \mathrm{~h}$ à $110^{\circ} \mathrm{C} ;{ }^{c}$ : selon Kayser et Patton (1970) : membrane du globule gras, transméthylation par $\mathrm{HCl}$-méthanol $18 \mathrm{~h}-24 \mathrm{~h}$ à $80^{\circ} \mathrm{C}$; $d$ : pourcentage relatif exprimé par rapport à la somme des acides gras rapportés dans le tableau. Pour l'intérêt de la comparaison, les sommes des acides gras provenant des références bibliographiques sont ramenées à 100.

Fatty acid composition of ceramide-hexosides obtained from different dairy fractions. Influence of hydrolysis. a: from Morrison et al (1965): buttermilk, $\mathrm{BF}_{3}$-methanol transmethylation at $100{ }^{\circ} \mathrm{C}$ for 90 min; $b$ : from Morrison and Hay (1965): buttermilk, saponification followed by methylation with $\mathrm{BF}_{3^{-}}$ methanol at $110^{\circ} \mathrm{C}$ for $24 \mathrm{~h}$; $\mathrm{c}$ : from Kayser and Patton (1970): fat globule membrane, transmethylation by $\mathrm{HCL}$-methanol at $80^{\circ} \mathrm{C}$ for $18 \mathrm{~h}-24 \mathrm{~h}$; d: relative percentage calculated from the sum of fatty acids reported in the table. For interest of comparison sums of fatty acids issued from bibliographical references were corrected to 100.

\begin{tabular}{|c|c|c|c|c|c|c|c|}
\hline Acides gras & $\begin{array}{l}C M H+C D H) \\
\text { de } P L P\end{array}$ & $C M H^{a}$ & $\begin{array}{l}\text { gluco- } \\
\text { céramides }^{b}\end{array}$ & $\begin{array}{l}\text { gluco- } \\
\text { céramides }^{c}\end{array}$ & $C D H^{a}$ & $\begin{array}{c}\text { Lacto- } \\
\text { céramides }^{b}\end{array}$ & $\begin{array}{l}\text { Lacto- } \\
\text { céramides }\end{array}$ \\
\hline $14: 0$ & 0,7 & 6,3 & - & 3,7 & 2,7 & 0,3 & 2,5 \\
\hline $16: 0$ & 12,2 & 29,8 & - & 10,9 & 25,0 & 7,7 & 13,4 \\
\hline $16: 1$ & 0,8 & 2,6 & 1,4 & 0,7 & 1,2 & - & 6,3 \\
\hline $18: 0$ & 10,1 & 11,1 & 13,7 & 4,1 & 16,2 & 3,3 & 6,5 \\
\hline $18: 1 n-9$ & 21,7 & 16,7 & 12,2 & 1,7 & 13,9 & 1,3 & 6,0 \\
\hline $18: 2 n-6$ & 3,7 & 2,7 & 2,0 & - & 2,3 & 0,2 & - \\
\hline $18: 3 n-3$ & 0,7 & 0,5 & - & - & - & - & - \\
\hline $20: 0$ & 0,5 & 0,6 & 0,9 & 0,8 & 1,6 & 1,1 & 3,5 \\
\hline $20: 1 n-9$ & 1,2 & 0,2 & - & - & 1,1 & - & - \\
\hline $20: 4 n-6$ & 0,5 & - & - & - & - & - & - \\
\hline $20: 5 n-3$ & 0,2 & - & - & - & - & - & - \\
\hline $22: 0$ & 15,1 & 5,4 & 17,0 & 17,5 & 9,3 & 24,9 & 13,5 \\
\hline $22: 5 n-3$ & 0,5 & - & - & - & 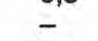 & - & - \\
\hline $23: 0$ & 18,9 & 8,6 & 22,0 & 23,1 & 12,5 & 29,5 & 16,9 \\
\hline $23: 1$ & 1,2 & 0,7 & 3,4 & 5,4 & - & 6,6 & 0,9 \\
\hline $24: 0$ & 11,2 & 5,1 & 9,4 & 15,8 & 7,1 & 16,5 & 10,7 \\
\hline $24: 1$ & 0,8 & 1,1 & 2,1 & 1,4 & 1,3 & 3,7 & 2,1 \\
\hline $25: 0$ & - & - & - & 2,0 & - & 0,7 & 1,9 \\
\hline Total AG \% & 100 & 91,4 & 84,1 & 87,1 & 94,2 & 95,8 & 84,2 \\
\hline AG saturés d & 68,7 & 73,2 & 75,0 & 89,3 & 79,0 & 87,5 & 81,9 \\
\hline$A G$ insaturés ${ }^{d}$ & 31,3 & 26,8 & 25,0 & 10,7 & 21,0 & 12,5 & 18,1 \\
\hline $\mathrm{AG} \mathrm{C} 20$ à $\mathrm{C} 25^{\mathrm{d}}$ & d 50,1 & 23,8 & 65,2 & 75,7 & 35,0 & 86,6 & 58,9 \\
\hline
\end{tabular}

L'hydrolyse de l'acide gras lié par une liaison amide au sein des sphingolipides nécessite des conditions plus vigoureuses que celles établies pour les glycérophospholipides. Nous avons constaté une augmentation importante des acides gras à nombre d'atomes de carbone supérieur à 20 quand la transméthylation est poursuivie $14 \mathrm{~h}$ à $90^{\circ} \mathrm{C}$. Déjà en 1964 , Patton et al avaient préconisé des temps de réaction de 18 à $24 \mathrm{~h}$ avec un mélange méthanolacide sulfurique $5 \%$, pour détecter valable- 
ment ces acides à longue chaîne. La composition en acides gras de la fraction céramides héxosides $(\mathrm{CMH}+\mathrm{CDH})$ de PLP peut être comparée à celle obtenue par plusieurs auteurs pour des céramides héxosides de différentes fractions laitières (tableau IV). II apparaît de grands écarts dans cette composition, principalement pour les acides gras à longue chaîne, les 2 séries de résultats de l'équipe de Morrison montrant bien l'influence de l'hydrolyse vis à vis de cette composition.

Pour des conditions de transméthylation similaires, nos valeurs révèlent un degré de saturation plus faible et une moindre proportion d'acides gras à longue chaîne (C20 à C25); cet écart est dû à la grande quantité d'acide oléïque présent dans nos échantillons. PLP provient de lait écrémé où Kayser et Patton (1970) ont noté que les céramides héxosides présentaient des teneurs plus importantes en C16:0, C16:1 et $\mathrm{C} 18: 1$, que ceux issus de la membrane du globule gras. Hormis cette différence pour les acides gras inférieurs à C20:0, le profil des céramides héxosides de PLP est très voisin de celui des lactocéramides de membrane du globule gras, rapporté par ces mêmes auteurs.

\section{CONCLUSION}

Les lipides de PLP présentent une répartition lipides neutres-lipides polaires comparable à celle donnée pour les lipides de la membrane du globule gras. Toutefois les lipides polaires présentent une distribution entre phospholipides et céramides héxosides différente de celle habituellement rencontrée. Selon Kayser et Patton (1970), les céramides héxosides représentent $11 \%$ des lipides polaires de cette membrane. Nous constatons donc que le processus technologique d'ultrafiltration s'est traduit par un enrichissement préférentiel de ces lipides, leur importance étant multipliée par 3.

Nos résultats montrent que les lipides les plus insaturés voient leur proportion diminuée ( $\mathrm{PE}$, par exemple). Les lipides constitués d'acides gras à chaînes longues, tels que SM et tout particulièrement les céramides héxosides, augmentent en quantité notable. Le processus d'ultrafiltration semble permettre une sélection de ces molécules à caractère lipophile très marqué, ces molécules étant les plus à même de s'ancrer, par liaison de type hydrophobe, aux protéines du lactosérum.

\section{REMERCIEMENTS}

Les auteurs remercient bien vivement le Laboratoire de recherches de technologie laitière de I'INRA de Rennes d'avoir fourni la poudre PLP.

\section{RÉFÉRENCES}

Ames BN (1966) Assay of inorganic phosphate, total phosphate and phosphatases. In: Methods in Enzymology, vol VIII. Academic Press, New York, 115-118

Christie WW (1982) Lipid Analysis. Pergamon Press, Oxford

Christie WW (1983) The composition and structure of milk lipids. In: Developments in Dairy Chemistry. 2. Lipids (Fox PF, ed) Applied Science Publishers, London, New York, 1-35

Fauquant J, Vieco E, Brule G, Maubois JL (1985) Clarification des lactosérums doux par agrégation thermo-calcique de la matière grasse résiduelle. Lait $65,1-20$

Fujishima T, Ito S, Negishi T, Fujino $Y$ (1970) Studies on the lipids of cheese I : on the composition of lipids and fatty acids in gouda cheese. Rakuno Kagaku No Kenkyu 19, A113-120

Kayser SG, Patton S (1970) The function of very long chain fatty acids in membrane structure: evidence from milk cerebrosides. Biochem Biophys Res Commun 41, 1572-1578 
Marmer WN, Maxwell RJ (1981) Dry column method for the quantitative extraction and simultaneous class separation of lipids from muscle tissue. Lipids 16, 365-371

Maubois JL, Pierre A, Fauquant $\mathrm{J}$, Piot $\mathrm{M}$ (1987) Industrial fractionation of main whey proteins. Bull IDF212, 154-159

Maxwell RJ, Mondimore D, Tobias J (1986) Rapid method for the quantitative extraction and simultaneous class separation of milk lipids. J Dairy Sci 69, 321-325

Morrison WR, Hay JD (1970) Polar lipids in bovine milk. II. Long chain bases, normal and 2-OH fatty acids, an isomeric cis and trans monoenoic fatty acids in the sphingolipids. Biochim Biophys Acta 202, 460-467

Morrison WR, Jack EL, Smith LM (1965) Fatty acids of bovine milk glycolipids and phospho- lipids and their specific distribution in the diacylglycerophospholipids. J Am Oil Chem Soc 42, 1142-1147

Patton S, Durdan A, Mc Carthy RD (1964) Structure and synthesis of milk fat. VI. Unity of the phospholipids in milk. J Dairy Sci 47, 489495

Skipski VP, Barclay M (1969) Thin layer chromatography of lipids. In: Methods in Enzymology, vol XIV. Academic Press, New York, 530598

Wolff RL, Combe N, Entressangles B (1984) Incorporation et élimination de l'acide élaïdique dans les différents phospholipides des mitochondries ou de leurs membranes chez le rat. Incidence de l'organe et de l'âge. Rev Fr Corps Gras 31, 161-170 